\title{
Utility of Statistical Model Checking of Stochastic Hybrid Automata for Patient Controlled Analgesia
}

\author{
Henrikas Pranevicius ${ }^{1}$, Darius Naujokaitis ${ }^{1}$, Vytautas Pilkauskas ${ }^{1}$, Osvaldas Pranevicius ${ }^{2}$, \\ Mindaugas Pranevicius ${ }^{3}$ \\ ${ }^{1}$ Department of Informatics, Kaunas University of Technology, \\ Studentu St. 50-407, LT-51368 Kaunas, Lithuania \\ ${ }^{2}$ Department of Anesthesiology, New York-Presbyterian/Queens Flushing, \\ USA \\ ${ }^{3}$ Albert Einstein College of Medicine, \\ NY, USA \\ darius.naujokaitis@ktu.lt
}

\begin{abstract}
Opiate concentration in the effect compartment of the brain (OCEC) determines both, the pain control and the side effects. This concentration can be estimated using pharmacodynamics models; however, these models do not predict OCEC when delivery of the drug is random.

We are proposing to use stochastic hybrid automata for the verification of individualized model of patient's drug demands and model of patient's pharmacokinetics for the estimation of OCEC, and to express results as the probability of falling below the minimum effective analgesic concentration (MEAC) and/or probability of exceeding toxic concentration threshold. Patient controlled analgesia (PCA) model was based on the stochastic hybrid automata, while the verification of the model was done using UPPALL-SMC tool. The suggested approach allowed for quantitative prediction of the OCEC.
\end{abstract}

Index Terms-Patient controlled analgesia; hybrid automata; verification; statistical model checking.

\section{INTRODUCTION}

When patient controlled analgesia (PCA) was originally introduced, the belief was that frequency of analgesic demand uniquely reflects the level of patient's pain [1]. Hence, the pain, a very subjective phenomenon, can essentially be formalized "by substitution" of its formalism with the formalism of PCA and by calculating achievable compartmental concentrations. That can be done by describing frequency of demand as a random process. At the same time the prescription can be viewed as an attempt to insure that delivered amount of the drug is sufficient to achieve effective concentration, yet at the same time avoiding side effects [2]. The drug delivery with PCA is highly variable and therefore estimation of the probability of drug falling below the minimum effective analgesic concentration $(M E A C)$ and/or exceeding toxic concentration threshold in the effect compartment cannot be done without the model of drug delivery.

Manuscript received 14 February, 2017; accepted 19 June, 2017.
There have been efforts made to address safety of the PCA devices utilizing formal verification approach by Sankaranarayanan et al., Arney et al. and Ruksenas et al. [3]-[5]. These attempts addressed multiple issues affecting safety of PCA pump, including mechanical plant, operator errors and patient pharmacokinetic differences; however neither of these studies accounted for drug demand variability originating from the individuality of each patient.

Kraujalis addressed the problem of drug delivery and distribution of drug concentration among various compartments using timed automata. However, the main problem with this approach was the necessity to express concentrations and amounts in real numbers: guards and predicates when using time automata formalism are carried out only with integer or rational, therefore real type of data had to be transformed into ranges. Then, using model checking technique for timed automata, the verification could be done using reachability analysis. However, if ranges are broad, an unmanageable "states explosion" occurs. In other words, it can be argued that application of the timed automata for verification of hybrid systems is not an appropriate formalism [6].

We are proposing a novel methodology, which can be used for exactly this purpose, predicting the drug concentration in the effect compartment during variable delivery of the drug. The variable delivery of the drug for individual patient is described using patient's behavior model as statistical $A R M A$ model, which allows to estimate and/predict the stochastic process of drug demand.

Such approach for the first time allowed to combine behavioral model of patient's analgesia demand, pharmacokinetic model (both described as a network of stochastic hybrid automata) with the automatic verification of the model, using UPPALL-SMC, that was developed by the researchers at the Aalborg University and Uppsala University [7], [8]. UPPAAL-SMC is a subsystem of UPPAAL which can be effectively used for hybrid systems 
simulation and verification, using stochastic hybrid automata (SHA) [9], [10]. Moreover, the tool UPPAAL-SMC can describe and simulate similar automata models like hybrid automata, price timed automata, or just regular timed automata. The tool also allows checking the properties of those models.

Moreover, since the tool UPPAAL-SMC can use real-type variables and does not require transformation of real variables into the integer or rational intervals, it is more versatile than the UPPAAL tool: it allows hybrid models to be accurately modelled and simulated. Moreover, the expansion of UPPAAL tool by adding formalism of stochastic hybrid automata, where clock rates can be arbitrarily changed using various constants and expressions involving other clocks, allows to define ordinary differential equations (ODEs) very effectively [11]. The reachability analysis using stochastic hybrid automata is decidable when applying statistical model checking (SMC) technique to the verification of system. The SMC is based on generation of random states trajectories of states graph. Those states trajectories are used to decide whether the system satisfies the property with some degree of confidence. Such application of SMC helps to avoid an exhaustive exploration of the state-space of the system model [8].

Thus UPPAAL-SMC allowed effective verification of our model, opening the possibility of verification of such clinically relevant qualities as the probability of effect concentration falling below the minimum effective analgesic concentration $(M E A C)$ and/or exceeding toxic concentration threshold, all with arbitrary selected confidence intervals [8].

\section{StOchastic Hybrid Automata Model}

\section{A. Hybrid Automata Model}

We used hybrid systems simulation and verification method based on the stochastic hybrid automata ( $S H A)$ formalism, which for the clarity purposes we are outlining here.

Hybrid automata $(H A)$ model is a special case of automaton models that is described by a tuple $H=\left(L, l_{0}, X, E, F, I\right)[12]$, where

$-L$ is a finite set of locations,

$-l_{0}$ is the initial location,

$-X$ is a finite set of continuous variables,

$-E$ is a finite set of edges where each edge is defined by suite $\left(l, g, a, \varphi, l^{\prime}\right)$, where

$l, l^{\prime} \in L$,

$g$ is a predicate on $R^{X}$,

$a$ represents an action,

$\varphi$ is the binary relation on $R^{X}$.

$-F$ is the delay function for each $l \in L$,

$-I$ is the invariant mapping that assigns invariant predicates $I(l)$ to locations, whit the assumptions that:

$a$ variable valuation is a mapping $v$ from the continuous variables $X$ to the real's $R, v: x \rightarrow R$. So $R^{X}$ represents a set of valuations over $X$,

the delay function $F$ represent evolution of the valuations on $X$ over time. Thus $F(d, v)$ is the new valuation on $X$, after time delay $d$.

Transition between states $(l, v)$ of $H A$ is dependent on a continuous value $d$ (delay function) and a discrete value $a$ (action) respectively; $(l, v) \stackrel{d}{\rightarrow}\left(l, v^{\prime}\right)$ and $(l, v) \stackrel{a}{\rightarrow}\left(l^{\prime}, v^{\prime}\right)$, where $l^{\prime}$ and $v^{\prime}$ represent valuations for new location and new valuations of variables must exist as an edge $e \in E$, must be available $v \vDash g$, and $\varphi\left(v, v^{\prime}\right)$.

Thus interconnected $H A$ are used for hybrid system modelling and the entire network is called the network of hybrid automata $(N H A)$. NHA is defined as tuple $A^{j}=\left(L^{j}, X^{j}, \Sigma^{j}, E^{j}, F^{j}, I^{j}\right)$, where $j=1 \ldots n$ is an index of n $H A$ on the network, $\Sigma=\Sigma_{i} \cup \Sigma_{o}$ is an action set of the inputs $\Sigma_{i}$ and outputs $\Sigma_{o}$, transition between separate $H A$, and $X^{j}, \Sigma^{j}, E^{j}, F^{j}, I^{j}$ are sets of locations, continuous variables, edges, delay functions and predicates on the $H A$ with index $j$ [11].

Sometimes hybrid systems depend on accidental events or distribution of their parameters. Therefore, we used $H A$ with the stochastic behavior: Stochastic hybrid automaton $(S H A)$ is a developed hybrid automaton, that has an additionally flexibility for $\mathrm{t}$ hybrid system representation. Stochastic behavior of $H A$ is represented by the non-deterministic delay function, output, or the next state [13], id est. if it exists:

- the delay density function $\mu_{S}$,

- the output probability function $\gamma_{S}$,

- the next-state density function $\eta_{s}$.

Therefore, when at least one hybrid automaton has stochastic behavior on $N H A$, then network of $H A$ could be determined as a network of stochastic hybrid automata (NSHA).

The delay density function $\mu_{s}$ has either uniform or exponential distribution depending on invariant of $l$. Denote the supremum delay by $D(l, v)$ and infimum delay by $d(l, v)$ before enabling an output. If $D(l, v)<\infty$ then the delay density function $\mu_{s}$ has uniform distribution on $[d(l, v), D(l, v)], \quad$ otherwise $\mu_{s} \quad$ is an exponential distribution with rate $P(l)$. The output probability function $\gamma_{s}$ is the uniform distribution for every state output probability function $s(l, v)$ [9].

\section{B. Statistical Model Checking}

We used hybrid system verification method, based on statistical model checking (SMC) technique to verify network of hybrid automata models: statistical model checking extends runtime verification capabilities and the result of SMC technique can give answers to whether or not hybrid system satisfies given property or hybrid system satisfies given property with a probability greater than some threshold. Difference between classical model checking and SMC technique is that the result of SMC technique is evaluated with preselected confidence $(95 \%, 99 \%$, etc.) [10]. 
The properties, which are there verified, are described by Weighted Metric Temporal Logic (WMTL). A WMTL formula $\varphi$ over the proposition $P$ and the clock $C$ is generated by the grammar

$$
\begin{gathered}
\varphi::=p|\neg p| \varphi|\neg \varphi| \varphi_{1} \wedge \varphi_{2}\left|\varphi_{1} \vee \varphi_{2}\right| O \varphi \mid \\
\left|\varphi_{1} \bigcup_{[a, b]}^{c} \varphi_{2}\right| \varphi_{1} R_{[a, b]}^{c} \varphi_{2},
\end{gathered}
$$

where $p \in P, a \leq b$ and $c \in C$.

For these formulas $\varphi$ are obtained from Boolean laws: Formula $\varphi$ means that $\varphi$ should be satisfied starting from the next observation of the run. Formula $\varphi_{1} \bigcup_{[a, b]}^{c} \varphi_{2}$ is satisfied by a run if $\varphi_{1}$ is satisfied on the run until $\varphi_{2}$ is satisfied, and this will continue while value of clock $c$ increases with more than $b$ starting from the beginning of the run, and after it increased for more than $a$. The release operator $R$ is dual to $\bigcup$, and

$$
\varphi_{1} R_{[a, b]}^{c} \varphi_{2} \equiv \neg\left(\neg \varphi_{1} \bigcup_{[a, b]}^{c} \neg \varphi_{2}\right),[14]
$$

We used statistical model checking tool UPPAAL-SMC, where model statistical checking technique is realized based on $W M T L_{\leq}$which is a subset of WMTL. The logic $W M T L_{\leq}$ is defined by the grammar

$$
\varphi::=p|\neg \varphi| \varphi_{1} \wedge \varphi_{2}|O \varphi| \varphi_{1} \bigcup_{\leq d}^{c} \varphi_{2}
$$

where $d \in N$. Formula $\varphi_{1} \bigcup_{\leq d}^{c} \varphi_{2}$ is satisfied by a run if $\varphi_{1}$ is satisfied on the run until $\varphi_{2}$ is satisfied, and this will continue while value of clock $c$ increases with more than $d$ [15].

The logic $W M T L_{\leq}$is applied for the evaluation of probability or making comparison between them, or to test hypothesis about properties $\varphi_{i}$. The probability $P_{A}(\varphi)$ is defined as the probability of the network of stochastic hybrid automata $\mathrm{A}$, that a random run of A satisfies property $\varphi$. Notation $\diamond_{\leq d}^{c} \varphi=$ true $\bigcup_{\leq d}^{c} \varphi$ means that $\varphi_{1}$ is satisfied on all runs until clock $c$ is lower than threshold $d$ and the satisfaction of property $\varphi$ is checked [10], [11] and [15].

The notation $\vartheta_{\leq d}^{c} \varphi$ is used on the statistical model checking algorithms of UPPAAL-SMC and can answer the following three types of questions: Hypothesis testing: Is the probability $P_{A}\left(\diamond_{\leq d}^{c} \varphi\right)$ for a given NSHA $A$ greater or equal to a certain threshold $p \in[0,1]$ ? Probability evaluation: What is the probability $P_{A}\left(\diamond_{\leq d}^{c} \varphi\right)$ for a given NSHA $A$ ? Probability comparison: Is the probability $P_{A}\left(\diamond_{\leq d}^{c} \varphi_{1}\right)$ greater than the probability $P_{A}\left(\diamond_{\leq y}^{x} \varphi_{2}\right)$ ?

Accordingly, on the UPPAAL-SMC tool, followings queries are used for those three types of questions: $[$ bound $](\varphi)>=p_{0}, \quad \operatorname{Pr}[$ bound $](\varphi), \quad \operatorname{Pr}[$ bound $]\left(\varphi_{1}\right)>=$
$>=\operatorname{Pr}[$ bound $]\left(\varphi_{2}\right)$, where bound defines how to bound the runs [10].

\section{PAtient CONTROLled ANAlgesia Model}

\section{A. Pharmacokinetic Model}

A three compartmental model of drug distribution between the plasma and the brain tissue (effect compartment) was used to describe fentanyl and morphine pharmacokinetics/pharmacodynamics; all of it is shown in Fig. 1.

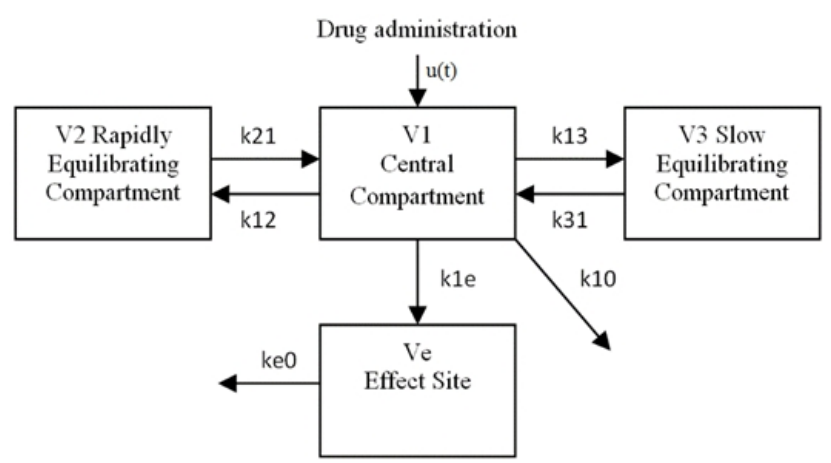

Fig. 1. Three compartment pharmacokinetic model.

The central compartment $\left(V_{1}\right)$ represents distribution volume. The peripheral compartments $\left(V_{2}, V_{3}\right)$ are composed of tissues and organs, where drug distributes at a rapid and slow rate. The effect site is the hypothetical compartment that relates the time course of plasma drug concentration to the time course of drug effect [16].

Pharmacokinetic model is described by four differential equations:

$$
\left\{\begin{array}{l}
d x_{1} / d t=k_{21} x_{2}+k_{31} x_{3}-\left(k_{12}+k_{13}+k_{10}\right) x_{1}+u(t), \\
d x_{2} / d t=k_{12} x_{1}-k_{21} x_{2}, \\
d x_{3} / d t=k_{13} x_{1}-k_{31} x_{3}, \\
d x_{e} / d t=k_{e 0} x_{1}-k_{e 0} x_{e},
\end{array}\right.
$$

where $x_{1}, x_{2}, x_{3}$ and $x_{e}$ are the amounts of drug in the central, second, third and effect site compartments, respectively, and $k_{10}, k_{12}, k_{13}, k_{21}, k_{31}$ and $k_{e 0}$ are the constants defining the elimination as well as intercompartmental transfer rates. The drug delivery function $u(t)$ is used to represent drug infusion control.

Scheme of patient model analgesia model is presented in Fig. 2.

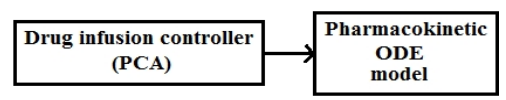

Fig. 2. Scheme of the PCA model.

\section{B. Patient's Behaviour Model}

We used two anonymous data logs (both exceeding 24 hour) from the Protocol Library Safety System, Moog, East 
Aurora, NY. One was with a prescription for morphine and another - for fentanyl. We analysed the length of time period (in minutes) between the two consecutive drug demands [17]. Although patient's demands for analgesia may be affected by a multiples factors, they could be reasonably approximated by the autoregressive moving average model of a stochastic process [18].

\section{1) Analysis of Morphine PCA Demands}

After comparing 25 different autoregressive moving average models, with parameter values of $p$ and $q$, ranging from 0 to 4 , we chose the model $\operatorname{ARMA}(p, q)$ with lowest value of Bayesian Information Criterion (BIC) is generated [17]. The analysis of the length of time period $y_{t}$ (in minutes) between the two consecutive drug requirements was identified, that $A R M A(0,0)$ has the lowest BIC value and it is suggested that the following model is optimal for morphine PCA demands

$$
y_{t}=\mu+k t+\varepsilon_{t}
$$

where $\varepsilon_{t} \sim W N\left(0, \sigma^{2}\right), \quad$ i.e., identically distributed independent white noise, with mean 0 and variation $\sigma^{2}$. Parameter $\mu$ is equal to 21,029 and $k$ is 0,407 .

Parameter estimates of this model suggest, that lag numerator $k$ does not significantly differ from 0 , therefore the time periods $y_{t}$ between two drug requirements during morphine analgesia can be simply modelled as: $y_{t}=\varepsilon_{t}$ where $\varepsilon_{t} \sim W N\left(0, \sigma^{2}\right)$. The time periods between the two drug demands are identically distributed and independent. Analysis of the $A R M A$ model noise $\varepsilon_{t}$ has shown it to have the exponential distribution with the parameter $\lambda=1 / 31.4$, which, according to the value of $\log$ likelihood of 222.34 , has provided the best fit [17].

Therefore, if $\mathrm{r}$ is a basic random number, the noise $\varepsilon_{t}$ could be generated by the exponential distribution as

$$
\varepsilon=-\ln r / \lambda
$$

\section{2) Analysis of Fentanyl PCA Demands}

For the simulation of the time period $y_{t}$, following model between two consecutive patient demands for fentanyl dose was selected

$$
y_{t}=11.57+0.264 t-0.302 y_{t-1}+0.203 y_{t-2}+\varepsilon_{t},
$$

where $\varepsilon_{t} \sim W N\left(0, \sigma^{2}\right)$ i.e., it is identically (though not necessary normally) distributed independent random variables, with mean 0 and variation $\sigma^{2}$.

Our analysis suggested that generalized distribution of the extreme values, that have parameters $k=-0.021633$, $s=16.521$ and $m=-9.426$ provided the best fit for $A R(2)$ model residual. Analysis was performed by maximizing the likelihood criterion. Chi square test did not reject our null hypothesis that residuals follow generalized extreme value distribution. Moreover, we compared residuals from fitted
$\operatorname{ARMA}(2,0)$ model with the data that was generated randomly and has generalized extreme values distribution with the same, already estimated, parameters. Distribution between residuals from ARMA and data which generated randomly was compared by means of Kolmogorov-Smirnov test - the result failed to reject the null hypothesis.

Thus, the value of random variable, having generalized extreme value distribution, can be generated by the following transformation of standard random number $r$ [17]

$$
\varepsilon=s \frac{(-\ln r)^{k}-1}{k}+m .
$$

\section{Sensitivity Analysis of ARMA Model}

The sensitivity analysis allows to identify model parameters whose inaccurate assessment would significantly reduce the accuracy of the model results. The sensitivity analysis of fentanyl ARMA model (7) was performed by varying the $A R M A$ model coefficients and by changing the standard deviation of the noise (9)

$$
S_{j}=1-\frac{\frac{1}{N} \sum_{i=1}^{N} y_{t i}^{j}}{\frac{1}{N} \sum_{i=1}^{N} y_{t i}},
$$

where $y_{t i}$ is the time period of fentanyl demand estimate by (4) and $y_{t i}^{j}$ - estimate, when varying $j$-th parameter ( $j=1,2,3)$. Sample size was $N=100$.

Results of sensitivity analysis are showed in Table I, where the first column is variation of the following parameters: coefficients $c_{1}, c_{2}$ and standard deviation $\sigma$. In the columns are presented percentage changes as measured by sensitivity index described by $S_{j}(9)$.

TABLE I. SENSITIVITY ANALYSIS OF FENTANYL ARMA MODEL.
\begin{tabular}{|c|c|c|c|}
\hline $\begin{array}{c}\text { Parameters } \\
\text { change }\end{array}$ & $\mathbf{S}_{\mathbf{1}}\left(\mathbf{c}_{\mathbf{1}}\right)$ & $\mathbf{S}_{\mathbf{1}}\left(\mathbf{c}_{\mathbf{1}}\right)$ & $\mathbf{S}_{\mathbf{1}}(\boldsymbol{\sigma})$ \\
\hline $1 \%$ & $0.27 \%$ & $0.18 \%$ & $0.83 \%$ \\
\hline $3 \%$ & $0.81 \%$ & $0.54 \%$ & $0.45 \%$ \\
\hline $5 \%$ & $1.35 \%$ & $0.89 \%$ & $2.93 \%$ \\
\hline $10 \%$ & $2.70 \%$ & $1.78 \%$ & $2.89 \%$ \\
\hline
\end{tabular}

The sensitivity analysis demonstrated that small changes in parameter values do not cause extreme changes in the model behaviour.

\section{Stochastic Hybid Automata of Patient CONTROLled ANALgesia Model}

\section{A. SHA of Pharmacokinetic Model}

The results of the implementation of the pharmacodynamics model in the UPPALL-SMC tool, using SHA formalism, are shown in Fig. 3, where ODE system of pharmacokinetic model is described as tool's function pharmODE().

Time durations Sup, Mup are estimated as total duration 
and maximal uninterrupted duration when concentration of drug of the effect compartment is higher than threshold Emax. In order to estimate Mup, an additional variable Tup is used.

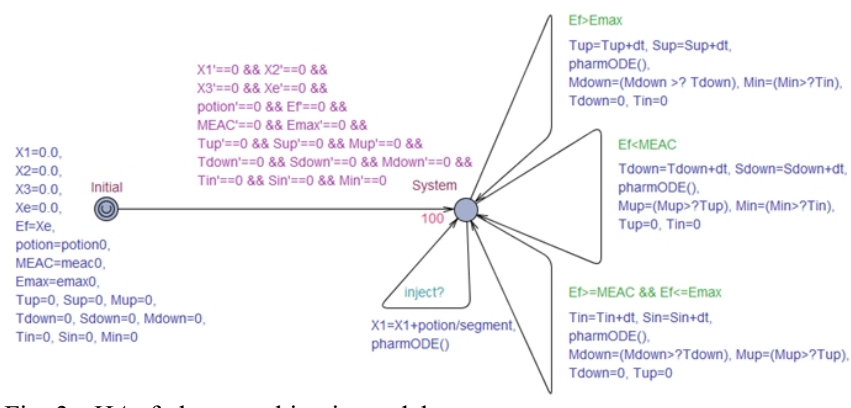

Fig. 3. $H A$ of pharmacokinetic model.

Hence, Sdown, Mdown and Tdown are the variables of time when concentration of drug in the effect compartment is lower than $M E A C$.

Variables Sin, Min and Tin represented duration when concentration of the drug in the effect compartment was acceptable, meaning that the drug concentration was higher than $M E A C$ and lower than Emax. The channel inject was used for the connection with pump to initialize virtual drug infusion.

\section{B. Drug Infusion Model (SHA Model)}

Developed model of patient controlled analgesia controls infusion of drug and has three states Initial, Injection and Sleep. Drug injection is made after the demand from the patient. Time duration $w$ represented the duration between two drug demands and was realized by the function $P B M()$. Function $P B M()$ varied depending on the type of drug and individual patient characteristics. Patient controlled analgesia model is shown in Fig. 4.

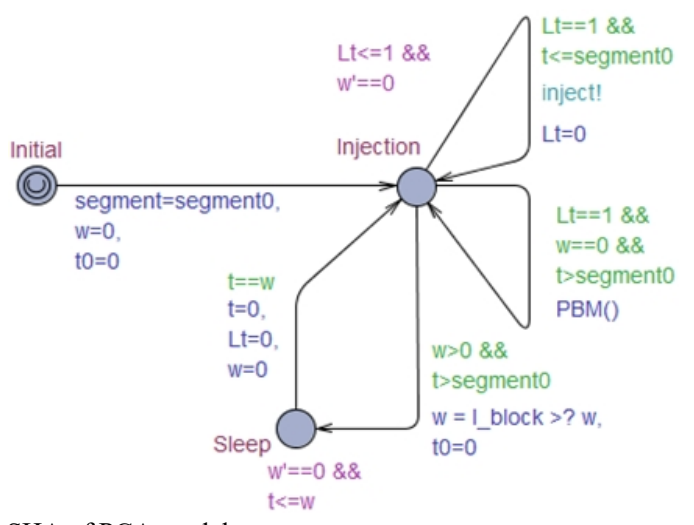

Fig. 4. SHA of PCA model.

We used $P B M()$ from earlier described $A R M A$ model. The duration of drug infusion was described by the variable segment0. Moreover, the PCA model included "lockout interval" which precluded repeat drug injection for I_block minutes. Therefore, if generated duration $\mathrm{w}$ was less than I_block minutes, the duration $\mathrm{w}$ was changed to I_block minutes.

Drug infusion dose and rate was simulated using: potion/segment parameters (Fig. 3).

On state Sleep the drug administration was not allowed.

Action inject! was communicated to the pharmacokinetic model and was set to transmit the signal for the start of drug infusion.

\section{Checking the Properties of PCA Model}

\section{A. Initial Conditions and Assumptions (Simulation Protocol)}

Simulation of morphine and fentanyl PCA was performed according to the aggregate scheme [19] as it is shown in the Fig. 1. Drug infusion controller simulated patient's demands, the probabilistic properties of which were defined by the $A R M A$ model that is described in Section II.

Pharmacokinetics of morphine and fentanyl was simulated by the three compartment model. The following parameters were used for the simulation of morphine pharmacokinetics:

Central compartment volume $=17.81$,

Time to deliver the bolus dose $=40 \mathrm{sec}$ (infusion rate for Moog, East Aurora, NY PCA pump), Bolus dose $=1 \mathrm{ml}$, Lockout interval $10 \mathrm{~min}$.

Morphine micro rate constants were chosen from [20].

For the simulation of fentanyl PCA, the following parameters were used:

Central compartment volume $=6.091$,

Time to deliver the bolus dose $=40 \mathrm{sec}$, Bolus dose $=$ $1 \mathrm{ml}$., Lockout interval $6 \mathrm{~min}$.

Fentanyl micro rate constants were chosen from Shafer publications [16].

\section{B. Model Properties Checking}

We checked seven properties of the PCA model for being "true" or "false". Duration of the simulation and checking was chosen to be 24 hours. We denoted $T_{i}^{u}$ as time intervals when concentration of drug in effect compartment $x_{e}$ is more than Emax. $T_{i}^{d}$ was denoted as time interval when concentration of drug in effect compartment is lower than $M E A C$. Then we described the checking properties of PCA model:

1. Is the total time less than $\tau_{h}=1$ hour, when concentration of drug in effect compartment $x_{e}$ is higher than $E \max$, i.e.: $\varphi_{1}: \sum_{i} T_{i}^{u}<\tau_{h}$ ?

2. Is the uninterrupted time less than $\tau_{m}=14.4$ minutes (i.e. $1 \%$ of checking time), when concentration of drug in effect compartment $x_{e}$ is higher than Emax, i.e.: $\varphi_{2}: \max _{i} T_{i}^{u}<\tau_{m} ?$

3 . Is the total time equal to the checking time ( 24 hours), when concentration of the drug in the effect compartment $x_{e}$ is higher than $M E A C$, i.e.: $\varphi_{3}: \sum_{i} T_{i}^{d}=24 \cdot \tau_{h}$ ?

4. Is the total time less than $\tau_{h}=1$ hour, when concentration of the drug in the effect compartment $x_{e}$ is lower than $M E A C$, i.e.: $\varphi_{4}: \sum_{i} T_{i}^{d}<\tau_{h}$ ?

5. Is the uninterrupted time less than $\tau_{m}=14.4$ minutes, when concentration of the drug in the effect compartment $x_{e}$ is less than $M E A C$, i.e.: $\varphi_{5}: \max _{i} T_{i}^{d}<\tau_{m}$ ?

6. Is the total time more than 12 hours, when the 
concentration of drug in the effect compartment $x_{e}$ is between $M E A C$ and Emax? i.e.: $\varphi_{6}: \sum_{i} T_{i}^{u}+\sum_{i} T_{i}^{d}<12 \cdot \tau_{h}$ ?

7. Is the total time more than $90 \%$ checking time $(0.9 \cdot 24$ hours), when concentration of the drug in the effect compartment $x_{e}$ is between $M E A C$ and Emax, i.e.: $\varphi_{7}: \sum_{i} T_{i}^{u}+\sum_{i} T_{i}^{d}<24 \cdot 0.1 \cdot \tau_{h} ?$

Those seven properties can be expressed as queries on UPPAAL-SCM tool using by $W M T L_{\leq}$logic. Accordingly, the notations $T_{i}^{u}$ and $T_{i}^{d}$ can be expressed by variables Mup and Mdown of SHA pharmacokinetic model on the Fig. 3. Variables Sup and Sdown are described using notations $\sum_{i} T_{i}^{u}$ and $\sum_{i} T_{i}^{d}$. The negative of sum $\sum_{i} T_{i}^{u}+\sum_{i} T_{i}^{d}$ corresponds to variable $\operatorname{Sin}$. Expressions of verifying properties $\varphi_{1}-\varphi_{7}$ on UPPAAL-SMC tool are shown in Table II.

TABLE II. PROPERTIES EXPRESSIONS ON UPPAAL-SMC

\begin{tabular}{|c|c|}
\hline Property & Expression on UPPAAL-SMC \\
\hline$\varphi_{1}$ & $\operatorname{Pr}[<=86400]([] \mathrm{Sup}<86400 / 24)$ \\
\hline$\varphi_{2}$ & $\operatorname{Pr}[<=86400]([] \mathrm{Mup}<86400 / 100)$ \\
\hline$\varphi_{3}$ & $\operatorname{Pr}[<=86400]([] \mathrm{Ef}>=\mathrm{MEAC})$ \\
\hline$\varphi_{4}$ & $\operatorname{Pr}[<=86400]([]$ Sdown $<86400 / 24)$ \\
\hline$\varphi_{5}$ & $\operatorname{Pr}[<=86400]([]$ Mdown $<86400 / 24)$ \\
\hline$\varphi_{6}$ & $\operatorname{Pr}[<=86400]([] \mathrm{Sin}>=86400 \cdot 0.5)$ \\
\hline$\varphi_{7}$ & $\operatorname{Pr}[<=86400]([] \mathrm{Sin}>=86400 \cdot 0.9)$ \\
\hline
\end{tabular}

\section{PCA Simulation}

Verification time of PCA model depends on the selected simulation/verification period. Some calculations took more than 2 hours when simulation/checking time was 24 hours.

Therefore, we made several steps to reduce computational time required for verification: we simulated ten stochastic runs of PCA model to identify time to reach the steady level of drug concentration in all compartments.

Figure 5 shows one stochastic run of PCA model with fentanyl (using UPPALL-SMC query: simulate $1 \quad[<=$ 86400] $\{S C r . X 1, S C r . X 2, S C r . X 3\})$ : green curve is fentanyl concentration in the slow equilibration compartment, yellow curve - drug concentration in the rapidly equilibrating compartment and red curve - in the central compartment.

Ten simulating runs of PCA model demonstrated that drug concentration reaches steady-state after about 2 hours' simulation (checking) time. Therefore, the initial conditions of PCA model could be replaced by the averages of variables at the end of second simulation hour. Modified initial state of PCA model is shown in Table III.

TABLE III. INITIAL STATE OF PCA MODEL.

\begin{tabular}{|c|c|c|}
\hline Compartment & Fentanyl & Morphine \\
\hline $\mathrm{x}_{1}$ & $1.274 \cdot 10^{-6}$ & $1.147 \cdot 10^{-6}$ \\
\hline $\mathrm{x}_{2}$ & $5.667 \cdot 10^{-6}$ & $5.666 \cdot 10^{-6}$ \\
\hline $\mathrm{x}_{3}$ & $4.758 \cdot 10^{-5}$ & $1.219 \cdot 10^{-5}$ \\
\hline $\mathrm{Xe}_{\mathrm{e}}$ & $1.225 \cdot 10^{-6}$ & $1.091 \cdot 10^{-6}$ \\
\hline
\end{tabular}

After the steady-state of a drug concentration is reached, it fluctuates around constant level. Therefore, the time for simulation and checking of the properties dropped down to 3 hours.

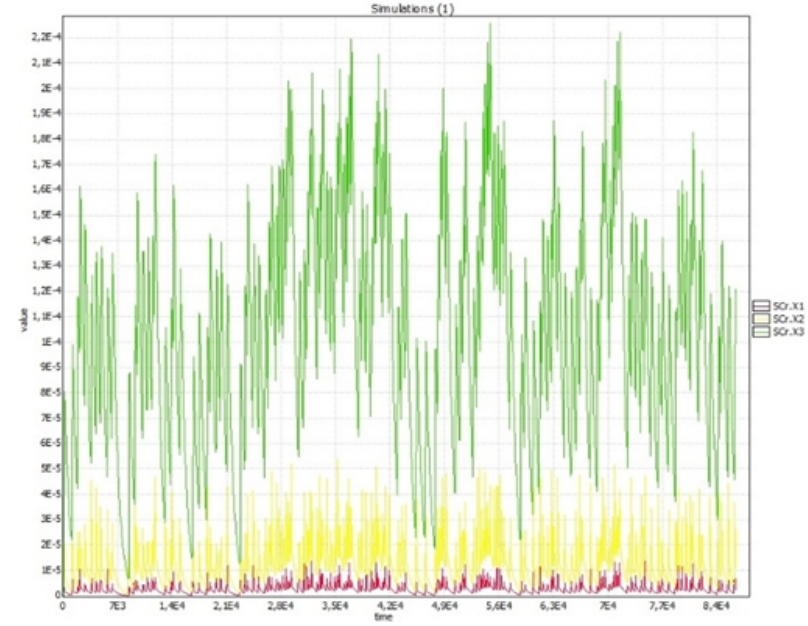

Fig. 5. One stochastic run of PCA model with fentanyl.

The simulation results of ten stochastic runs of PCA model for fentanyl using steady state modification of the initial state (from Table III) are shown in Fig. 6.

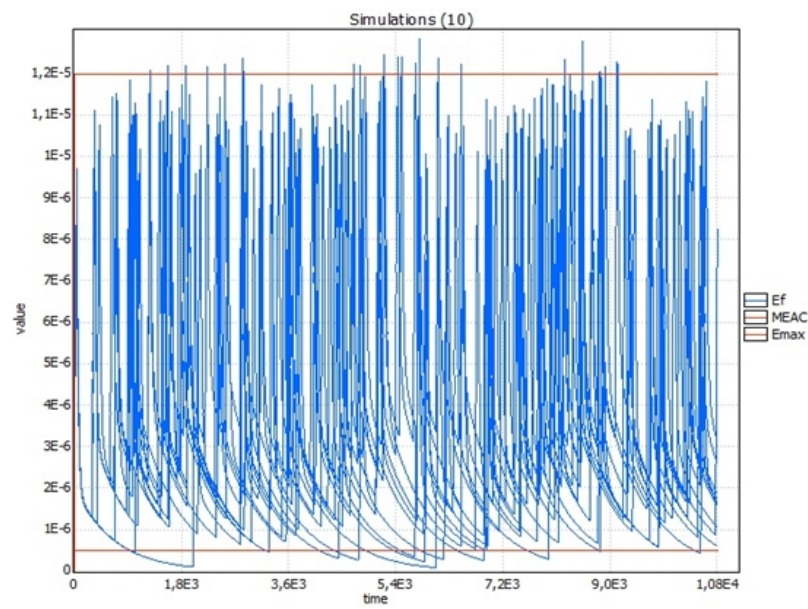

Fig. 6. Fentanyl concentration in the effect compartment with $M E A C$ and Emax shown for fentanyl (10 runs, 3 hours).

In Fig. 7 is shown one run of the drug concentration $x_{e}$ for three hours of simulation when modified initial conditions were used.

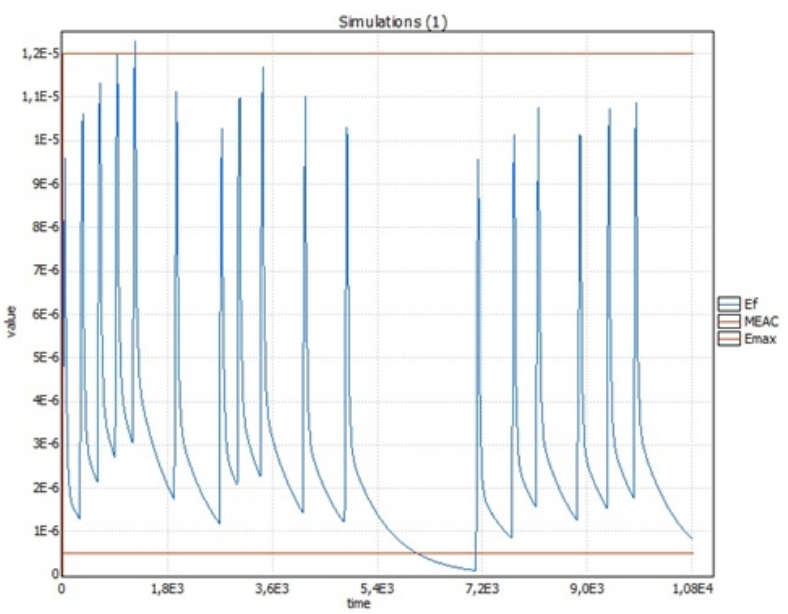

Fig. 7. Simulation run showing drug fentanyl concentration. 
In Fig. 7 Fentanyl concentration in the effect compartment indicates that concentration of drug crosses selected threshold (MEAC or Emax) several times. Besides, the interval, when drug concentration is outside of the interval between $M E A C$ and Emax, is variable.

\section{VERIFICATION RESULTS}

We verified all seven PCA model properties using steady state values for the initial state with total checking time of three hours.

Properties $\varphi_{1}-\varphi_{7}$ from the list of chapter 5.2 for fentanyl and morphine are shown in Table IV and in Table V. In both tables: "Time used for verification" represent calculation time for checking, "Runs" represent simulation count and "Probability interval" represent interval of estimating probability with $95 \%$ confidence interval, that property is satisfied.

TABLE IV. CHECKING PROPERTIES FOR FENTANYL PCA.

\begin{tabular}{|c|c|c|c|}
\hline Property & $\begin{array}{c}\text { Time used } \\
\text { verification }\end{array}$ & Runs & Probability interval \\
\hline$\varphi_{1}$ & $650 \mathrm{~s}$ & 36 & {$[0.903,1]$} \\
\hline$\varphi_{2}$ & $600 \mathrm{~s}$ & 36 & {$[0,903 ; 1]$} \\
\hline$\varphi_{3}$ & $600 \mathrm{~s}$ & 36 & {$[0,903 ; 1]$} \\
\hline$\varphi_{4}$ & $1900 \mathrm{~s}$ & 111 & {$[0,874 ; 0,974]$} \\
\hline$\varphi_{5}$ & $1350 \mathrm{~s}$ & 88 & {$[0,888 ; 0,987]$} \\
\hline$\varphi_{6}$ & $400 \mathrm{~s}$ & 36 & {$[0,903 ; 1]$} \\
\hline$\varphi_{7}$ & $980 \mathrm{~s}$ & 88 & {$[0,888 ; 0,987]$} \\
\hline
\end{tabular}

Table IV indicates that Fentanyl PCA with probability $>0.87$ satisfies all seven properties from chapter 5.2.

TABLE V. CHECKING PROPERTIES FOR MORPHINE PCA.

\begin{tabular}{|c|c|c|c|}
\hline Property & $\begin{array}{c}\text { Time used } \\
\text { verification }\end{array}$ & Runs & $\begin{array}{c}\text { Probability } \\
\text { interval }\end{array}$ \\
\hline$\varphi_{1}$ & $270 \mathrm{~s}$ & 36 & {$[0,903 ; 1]$} \\
\hline$\varphi_{2}$ & $300 \mathrm{~s}$ & 36 & {$[0,903 ; 1]$} \\
\hline$\varphi_{3}$ & $4500 \mathrm{~s}$ & 396 & {$[0,390 ; 0,490]$} \\
\hline$\varphi_{4}$ & $700 \mathrm{~s}$ & 97 & {$[0,884 ; 0,983]$} \\
\hline$\varphi_{5}$ & $1300 \mathrm{~s}$ & 175 & {$[0,829 ; 0,929]$} \\
\hline$\varphi_{6}$ & $650 \mathrm{~s}$ & 54 & {$[0,901 ; 0.999]$} \\
\hline$\varphi_{7}$ & $2350 \mathrm{~s}$ & 230 & {$[0,780 ; 0,880]$} \\
\hline
\end{tabular}

Table V. Morphine PCA satisfies six of seven properties determining with the probability $>=0.78$. The third property is not satisfied: it means that the total time period, when concentration of the drug in the effect compartment $x_{e}$ is higher than $M E A C$, is shorter than $100 \%$, but longer than $90 \%$ (seventh property shows that $90 \%$ of simulation time, $x_{e}$ is between $M E A C$ and toxic threshold).

\section{DISCUSSION}

Earlier research suggests, that different people show different demand patterns when using PCA [1], [21]. This behavioural pattern depends on a variety of simultaneously occurring factors: the level of pain, drug concentration in plasma and in the effect site, various side effects (e.g., nausea, sedation, respiratory depression), or even the psychological state of the patient, such as anxiety, cognitive impairment or sleep [2]. All these simultaneous factors introduce randomness in the demand pattern [22]. Therefore, demand sequence could be thought of as a random process that allows to use auto-regression moving average model ( $\operatorname{ARMA}(p, q))$ which is commonly used in the time series forecasting [18]. Depending on the different type of drugs being used, ARMA model exhibits variability of its parameters $p$ and $q$ [17].

Once acquired, ARMA behavioural model can be combined with the hybrid automata pharmacokineticpharmacodynamics model for simulation and estimation of the drug concentration in the effect compartment. This approach allowed predicting of the probability that drug concentration in the effect compartment will fall below the predetermined $M E A C$ (minimal effective analgesic concentration) and/or will exceed Emax (predetermined concentration for the side effects of the drug).

The proposed methodology allows to combine behavioural model of the patient with the formalism of pharmacodynamics model and thus to predict drug concentration variation in the effect-compartment in each individual patient.

Main value of this approach is the ability to formally check the stochastic variations in plasma and effect compartment concentrations in each individual patient against experimentally established populational norms for minimal effective and toxic concentration. If populational norms for the given drug are not available, the proposed approach allows their estimation from the larger sample of PCA logs.

Furthermore, this approach can be used not only for a single drug, but also for the combination of medications, such as morphine/fentanyl as it was used by Friedman [23]. When experimentally determining interaction at different ratios is not practical or feasible, the model based checking may be used to find optimal combination.

$M E A C$ and Emax values in this analysis were chosen arbitrarily from the populational data. To determine individual $M E A C$ and Emax (in addition to pump log) we would need more data, namely the information about the level of pain and the degree of respiratory depression. However, even with these limitations, combining pharmacokinetic and patient behavioural model allowed to estimate probabilities and average duration of intervals when drug concentration exceeds populational norms for analgesia and toxic effect thresholds. Described simulation and verification system for the patient controlled analgesia allows probabilistic prediction of the drug concentration that reaches and exceeds predetermined population threshold. This can be used for populational analysis of PCA prescription and to form the basis of a standardized drug library.

In this study we did not perform systematic analysis of ARMA patient demand models and thus did not determine parameter variability between different patients and between different days of PCA therapy. Although it would require larger data set, hybrid simulation method that was used here makes such analysis feasible.

Moreover, the methodology that was used to evaluate patient controlled analgesia performance avoids "explosions 
of states" that otherwise might have occurred when using time automata verification. In summary, statistical model checking verified the stochastic hybrid automata based analgesia model and allowed statistical prediction of the opiate concentration in the effect compartment.

\section{CONCLUSIONS}

We developed stochastic hybrid automata model and automatic model checking procedure that checks for the concentrations of two drugs in the effect compartment given the personalized pattern of patient demand for these drugs. This can be used for in silico evaluation of pharmacodynamics effects of customary compounded drug mixture, for guiding the transition to oral medication and for drug tapering and rotation.

Modelling results suggest that periods above critical (toxic) concentration threshold of morphine are less frequent, but of a longer duration as compared to fentanyl.

While clinical comparison did not detect difference in the side effects and effectiveness between morphine and fentanyl PCA [24], more frequent dose adjustments were needed for fentanyl PCA. This is consistent with our verification results that demonstrate larger variation in fentanyl concentration.

Special clinical application of this approach allows the investigation infusion of drug mixtures [23] (such as morphine/fentanyl; morphine/alfentanyl; morphine/ketamine for patient controlled analgesia) that can easily cause a variety of the desirable and nefarious side effects simultaneously.

Of note, in some patients $M E A C$ may exceed toxic concentration, leading to analgesia/sedation mismatch, when patient demonstrates toxic effects and inadequate analgesia at the same time. And although individual $M E A C$ and toxic concentration thresholds were not addressed in this study, it is certainly possible to do such estimation. For this purpose, data about pain ratings and side effects, such as respiratory depression, has to be available.

Side effects are random variable which is conditioned on concentration; and although it is true that analgesia and side effects cannot be determined solely by the concentration of the drug, it is also true that without the estimation of the concentration of the drug, no statement can be made about drug effectiveness and the potential for side effects in the particular patient.

Once populational normative for $M E A C$ and toxic concentration are established, model based verification will allow to determine the patients that will need higher monitoring acuity, institution of continuous positive pressure and/or decrease of the PCA dose. As such, the methodology described in this article can be used to standardize PCA prescription using populational data.

\section{REFERENCES}

[1] J. A. Grass, "Patient-controlled analgesia", Anesthesia \& Analgesia, vol. 101, pp. 44-61, 2005. [Online]. Available: http://dx.doi.org/10.1213/01.ANE.0000177102.11682.20

[2] M. Boom, E. Olofsen, M. Neukirchen, R. Fussen, J. Hay, G. J. Groeneveld, L. Aarts, E. Sarton, A. Dahan, "Fentanyl utility function", Anesthesiology, vol. 119, no. 3, pp. 663-674, 2013.
[Online]. Available: http://dx.doi.org/10.1097/ALN.0b013e31829 ce $4 \mathrm{cb}$

[3] S. Sankaranarayanan, H. Homaei, C. Lewis, "Model-based dependability analysis of programmable drug infusion pumps", Springer Science+Business Media, Lecture Notes in Computer Science, pp. 317-334, 2011. [Online]. Available: https://doi.org/ 10.1007/978-3-642-24310-3_22

[4] D. Arney, R. Jetley, P. Jones, I. Lee, O. Sokolsky, "Formal methods based development of a PCA infusion pump reference model, generic infusion pump (GIP) project", Joint Workshop on High Confidence Medical Devices, Software, and Systems and Medical Device Plug and-Play Interoperability, (HCMDSS-MDPnP 2007), 2007. [Online] Available: https://doi.org/10.1109/HCMDSS-MDPnP.2007.36

[5] R. Ruksenas, P. Curzon, A. Blandford, J. Back, "Combining human error verification and timing analysis: a case study on an infusion pump", Formal Aspects of Computing, vol. 26, no. 5, pp. 1033-1076, 2013. [Online]. Available: https://doi.org/10.1007/s00165-013-02881

[6] T. Kraujalis, "Modeling and verification of fuzzy control systems using Piece linear aggregate formalism", PhD dissertation, Inst. Math. Inf., Vytautas Magnus Univ. \& Vilnius Univ., 2014.

[7] G. Behrmann, A. David, K. G. Larsen, P. Pettersson, W. Yi, "Developing UPPAAL over 15 years", Software: Practice and Experience, vol. 41, no 2, pp. 133-142, 2011. [Online]. Available: http://dx.doi.org/10.1002/spe.1006

[8] A. David, K. G. Larsen, A. Legay, M. Mikucionis, D. B. Poulsen, "Uppaal SMC tutorial", International Journal on Software Tools for Technology Transfer, vol. 17, no. 4, pp. 397-415, 2015. [Online] Available: https://doi.org/10.1007/s10009-014-0361-y

[9] A. David, K. G. Larsen, A. Legay, M. Mikucionis, D. B. Poulsen, J Vliet, Z. Wang, "Statistical model checking for networks of priced timed automata", Lecture Notes in Computer Science, pp. 80-96, 2011. [Online]. Available: https://doi.org/10.1007/978-3-642-243103

[10] P. Bulychev, A. David, K. G. Larsen, M. Mikucionis, D. B. Poulsen, A. Legay, Z. Wang, "UPPAAL-SMC: Statistical Model Checking for Priced Timed Automata", Electronic Proceedings in Theoretical Computer Science, vol. 85, pp. 1-16, 2012. [Online]. Available: http://dx.doi.org/10.4204/EPTCS.85.1

[11] A. David, D. Du, K.G. Larsen, A. Legay, M. Mikucionis, D. B. Poulsen, S. Sedwards, "Statistical model checking for stochastic hybrid systems", Electronic Proc. Theoretical Computer Science, vol. 92, pp. 122-136, 2012. [Online]. Available: http://dx.doi.org/10.4204/EPTCS.92.9

[12] T. A. Henzinger, "The theory of hybrid automata", in Proc. 11th Annual IEEE Symposium on Logic in Computer Science, 1996 pp. 1-30. [Online]. Available: http://dx.doi.org/10.1109/ LICS.1996.561342

[13] A. David, K. G. Larsen, A. Legay, D. B. Poulsen, "Statistical model checking of dynamic networks of stochastic hybrid automata", Electronic Communications of the EASST, vol. 66, 2013. [Online] Available: http://dx.doi.org/10.14279/tuj.eceasst.66.893

[14] P. Bulychev, A. Davi, K.G. Larsen, A. Legay, G. Li \& D.B. Poulsen, "Rewrite-Based Statistical Model Checking of WMTL, Runtime Verification", Springer Science+Business Media, 260-275.

[15] P. Bulychev, A. David, K. G. Larsen, A. Legay, G. Li, D. B. Poulsen, A. Stainer, "Monitor-based statistical model checking for weighted metric temporal logic", Int. Conf. Logic for Programming, Artificial Intelligence, And Reasoning, 2012, pp. 168-182. [Online]. Available: https://doi.org/10.1007/978-3-642-28717-6 15

[16] S. L. Shafer, J. R. Varvel, N. Aziz, J. C. Scott, "Pharmacokinetics of fentanyl administered by computer-controlled infusion pump", Anesthesiology, vol. 73, no. 6, pp. 1091-1102, 1990. [Online] Available: https://doi.org/10.1097/00000542-199012000-00005

[17] H. Pranevicius, M. Snipas, T. Kraujalis, M. Pranevicius, O. Pranevicius, V. Pilkauskas, "Probabilistic behavioral model for the detection of criticalities when using morphine and fentanyl PCA", 26th European Modeling and Simulation Symposium (EMSS 2014), 2014.

[18] S. Makridakis, M. Hibon, "ARMA models and the Box-Jenkins methodology", Journal of Forecasting, vol. 16, no. 3, pp. 147-163, 1997. [Online]. Available: http://dx.doi.org/10.1002/(SICI)1099131X(199705) 16:3<147::AID-FOR652>3.0.CO;2-X

[19] L. D'Errico, M. Loreti, "A process Algebra Approach to Fuzzy Reasoning", in Proc. of IFSA/EUSFLAT Conf., 2009, pp. 11361141.

[20] B. E. Dahlstrom, L. K. Paalzow, G. Segre, A. J. Agren, "Relation 
between morphine pharmacokinetics and analgesia", Journal of Pharmacokinetics and Biopharmaceutics, vol. 6, no. 1, pp. 41-53, 1978. [Online]. Available: https://doi.org/10.1007/BF01066062

[21] F. J. Overdyk, "Postoperative opioids remain a serious patient safety threat", Anesthesiology, vol. 113, pp. 259-260, 2010. [Online] Available: http://dx.doi.org/10.1097/ALN.0b013e3181e2c1d9

[22] A. Woodhouse, "The minimum effective concentration of opioids: A revisitation with patient controlled analgesia fentanyl", Regional Anesthesia and Pain Medicine, vol. 25, no. 3, pp. 259-267, 2000 [Online]. Available: https://doi.org/10.1097/00115550-20000500000009
[23] Z. Friedman, R. Katznelson, S. R. Phillips, C. Zanchetta, O. I. Nistor, L. B. Eisen, N. Siddiqui, "A randomized double-blind comparison of a morphine-fentanyl combination vs. morphine alone for patientcontrolled analgesia following bowel surgery", Pain Practice, vol. 8, no. 4, pp. 248-252, 2008. [Online]. Available: http://dx.doi.org/10.1111/j.1533-2500.2008.00212.x

[24] P. R. Howell, D. R. Gambling, T. Pavy, G. McMorland, M. J. Douglas, "Patient-controlled analgesia following caesarean section under general anaesthesia: a comparison of fentanyl with morphine", Canadian Journal of Anaesthesia, vol. 42, no. 1, pp. 41-45. [Online]. Available: http://dx.doi.org/10.1007/BF03010570 